\title{
DIBUJAR EN IDIOMAS. VISUALIZAR LA COLONIZACIÓN LINGÜÍSTICA EN EL SUDESTE ASIÁTICO
}

\author{
DRAW IN TONGUES. VISUALIZING LINGUISTIC COLONIZATION \\ IN SOUTHEAST ASIA \\ Cristina NUALART \\ GIA (Grupo de Investigación Asia) \\ Universidad Complutense de Madrid \\ cnualart@faculty.ie.edu
}

\begin{abstract}
Resumen: No faltan en las artes visuales creaciones que han integrado en su plástica el lenguaje oral, la escritura o la traducción, si bien no es tan frecuente que el lenguaje sea el sujeto de la obra. Ese es el caso de diversas obras realizadas durante la década de 1990 en antiguas colonias del Sudeste Asiático, para poner en cuestión la herencia cultural constituida por las palabras y los sistemas de escritura. Desde sus respectivos contextos, Vietnam y Singapur, dos artistas aportan un incisivo comentario sobre los usos politizados de la escritura, las lenguas vernáculas y la alfabetización. Piezas performativas del artista Truong Tan y de la artista Amanda Heng aportan nuevos modos de comprender el funcionamiento del lenguaje y de la violencia ejercida a través de la colonización lingüística.
\end{abstract}

Palabras clave: Colonización lingüística. Traducción. Sudeste Asiático. Artes Visuales. Performance.

\begin{abstract}
There is no shortage of artworks that have integrated the spoken word, writing or translation into their aesthetic form, although it is rare for language to be the subject of the works. This is the case of several works made during the 1990s in former colonies of Southeast Asia. The works call into question the cultural heritage that words and writing systems constitute. From their respective contexts, Vietnam and Singapore, two artists offer an incisive commentary on the politicized uses of vernacular languages and literacy. Performance pieces by artists Truong Tan and Amanda Heng contribute new ways of understanding the functioning of language, and the violence that can be exerted through linguistic colonization.
\end{abstract}

Keywords: Linguistic colonization. Translation. Southeast Asia. Visual Arts. Performance. 


\section{INTRODUCCIÓN}

Los sistemas de escritura han permitido fijar la oralidad, pero también han sido usados como un medio de expresión plástica (Méndez Baiges, 2017). La historia del arte ha dado merecido reconocimiento a creaciones con palabras, como por ejemplo la poesía visual o los collages de recortes de prensa, en el caso las primeras vanguardias artísticas principios del siglo XX. Es conocida asimismo la admiración por parte de artistas europeos de las calidades dibujísticas de la escritura caligráfica de Asia (Fundación Juan March, 2018). El presente artículo, sin embargo, propone un acercamiento al lenguaje y la escritura como sujetos de las obras plásticas, ese decir, como objetos de estudio de la investigación artística y de la creación visual.

Esta indagación nos aproxima a su vez a un contexto geográfico de gran diversidad cultural y lingüística, el Sudeste Asiático, escasamente representado en la alta divulgación hispana. Al menos desde la fundación de $\mathrm{ASEAN}^{1}$ en 1967, se ha querido promover una visión regional unificada, según la cual las diferencias lingüísticas, políticas y sistémicas características de cada nación se entienden como expresiones diversas de unos valores comunes a la región. Llegada la década de 1990, sin embargo, parecía evidente que el énfasis en un imaginario regional de la cultura no había conseguido conectar las artes de los diversos países tanto como se deseaba (Whiteman, Abdullah, Low y Scott, 2018).

El historiador de arte singapurense T. K. Sabapathy (1996) observaba que la historia del arte del Sudeste Asiático se circunscribía a las delimitaciones nacionales, y que en pocas ocasiones se ponían en relación obras de distintos países del entorno. Sabapathy (1996: 13) cita una ponencia de Marian Pastor Roces realizada en el marco de la segunda trienal artística regional, Asia Pacific Triennial, en la cual esta curadora filipina abogaba con pasión por la necesidad de usar palabras, rigurosamente y con propósito, en rechazo de los sistemas dominantes de representación, insuficientes para abordar el arte de otros contextos. Roces proponía pensar una "terminología calibrada" que respetase las culturas que han estructurado influencias foráneas en un sistema de significado que ha sobrevivido violentos encuentros con las hegemonías globales, pero que también permitiese inscribir las extinciones culturales, víctimas de la "máquina moderna", para facilitar el duelo y legitimar estas pérdidas (Sabapathy, 1996: 13). Más recientemente, Legaspi-Ramirez (2018) considera acertado ampliar los discursos regionales prestando más atención al rol de los afectos en el encuentro con las obras de arte, y formulando una historia del arte del Sudeste asiático que no se centre en preguntar qué significan las obras de arte, sino que cuestione cómo significan.

Por todo ello, este artículo pone en relación piezas del artista vietnamita Truong $\operatorname{Tan}^{2}$ y de la artista singapurense Amanda Heng ${ }^{3}$, examinando lo que significan las obras en

\footnotetext{
${ }^{1}$ Asociación de Naciones del Sudeste Asiático (en inglés: Association of Southeast Asian Nations).

${ }^{2}$ El nombre Trương Tân (aquí con sus tildes diacríticas), sigue el orden de apellido primero y nombre de pila al final, como es habitual en Vietnam y en otros lugares de Asia. Por ello, en las referencias bibliográficas no se usan comas entre nombre y apellidos para autores vietnamitas.

${ }^{3}$ Nombre completo Amanda Heng Liang Ngim, aunque se la conoce como Amanda Heng (Koh, 2014).
} 
sus diferentes contextos marcados por la dominación extranjera, así como argumentando que los métodos de transmisión de significado empleadas en estas creaciones son coherentes con las reflexiones recientes sobre la historia del arte regional.

El peso de la herencia cultural de las palabras y los sistemas de escritura se hacen tangibles en la selección artística aquí propuesta, que contrasta performances realizadas a finales del siglo XX, de las que emergen unos incisivos mensajes críticos sobre la colonización lingüística y los usos politizados de la escritura, las lenguas vernáculas y la alfabetización.

El punto de partida está dedicado a los sistemas de escritura, por lo que se perfila el contexto histórico del territorio actualmente llamado Vietnam, como prefacio a la práctica artística de Truong Tan. El artista concibe una performance que arremete contra la educación artística colonial. La obra incorpora recursos como el dibujo o la ubicación urbana, que alimentan la carga crítica, si bien es de especial relevancia el ingenioso uso de la traducción fonética como recurso contestatario.

Una segunda parte del análisis aborda la violencia lingüística causada por políticas culturales en Singapur. La artista Amanda Heng, singapurense de ascendencia étnica china, es testigo de que la occidentalización forzada ha impactado la oralidad y la comunicación cotidiana. No se puede subestimar la aportación de las obras de Heng a los feminismos globales, si bien nuestra atención se dirige a las voces de diversas culturas que tienen cabida en su práctica artística.

Finalmente, se analizan los puntos de conexión semiótica entre los modos de operar utilizados en las diversas obras.

\section{REESCRIBIR LA HERENCIA COLONIAL DE VIETNAM}

\subsection{Logogramas, escritura y alfabetización}

Las palabras han tenido expresión visual por lo menos desde que nacen los sistemas de escritura, en sí mismos extraordinarios recursos para construir o reconstruir la historia social. Un diccionario chino-vietnamita de datación incierta, pero posiblemente coincidente con el auge del Barroco en España, permite a John Phan (2013) desarmar diversos argumentos usados históricamente para dar una posición de privilegio a la escritura como tecnología civilizadora, frente a su supuesta ausencia en pueblos bárbaros o periféricos. El fascinante estudio de Phan invita a no desestimar el funcionamiento de la escritura porque las grafías tienen distinto impacto, según representen sonidos o conceptos. Phan explora el nom, el primer sistema desarrollado para escribir vietnamita.

El [chĩu nôm, 喃, 喃, o 字喃, literalmente el habla común, ha sido diversamente reclamado como sistema clásico, tradicional o nacional. Esta escritura semantosilábica estaba plenamente extendida durante la dinastía Ly (1010-1225 e.c.), pero en distintos momentos desde entonces ha sido objeto de politizados esfuerzos para darle un valor nacionalista, en base a su expresión lógica y sencilla de la lengua vernácula. Por ejemplo, 
el emperador Gia Long apoyó el uso del nom a lo largo de su reinado en el siglo XIX, si bien su sucesor Minh Mang hizo todo lo contrario y lo desincentivó, exigiendo un retorno al uso de ideogramas chinos (Thompson, 2010).

La escritura en nom ha desaparecido de todo plan educativo actual, aunque la obra cumbre de la literatura de Vietnam ${ }^{4}$, la epopeya Kim Van Kieu fue escrita en nom por Nguyen Du (2013) hacia finales del siglo XVIII ${ }^{5}$. En ese tiempo coexistían en Vietnam tres sistemas de escritura: el chino clásico (hán-văn), cuyos ideogramas habían sido la norma desde el primer periodo de dominio chino sobre Vietnam (43-939 e.c.), el nom y además el incipiente quoc ngu (quốc ngũu), una escritura basada en el alfabeto latino, a veces llamada demótica o vernácula.

Misioneros católicos habían diseñado las bases del quoc ngu en el siglo XVII, pero la alfabetización masiva de la ciudadanía no despegó hasta mucho después, cuando la ocupación francesa estaba firmemente consolidada. La enseñanza colonial implementada en Vietnam popularizó el uso del quoc ngu, sistema de escritura que prevalece en la actualidad. Hasta la década de 1910, el chino clásico era la escritura oficial, usada por intelectuales vietnamitas a lo largo del primer cuarto del siglo XX (Vinh 1995). Mientras tanto, aumentó la población capaz de leer y escribir el quoc ngu, en parte gracias a la facilidad de memorizar las pocas letras del alfabeto romano, frente a los cientos de caracteres de alfabetos en ideogramas que se necesitaba retener. Crecía el índice de alfabetización a la par que aumentaba el vocabulario de los anamitas. El vietnamita pertenece a la familia de las lenguas mon-khmer, aunque contiene algunos términos provenientes de la familia de lenguas tai, así como una alta proporción de préstamos del chino adquiridos durante dos mil años. Si bien el aprendizaje del francés no alcanzó ni mucho menos a la mayoría de la población indochina durante la época colonial, la influencia de esta lengua fue señera entre escritores francoparlantes que pudieron leer a autores como Victor Hugo. Por su parte, el movimiento anticolonial no tardó en impulsar la educación de masas, organizando clases hasta en las zonas más remotas, recurriendo incluso a profesorado que a veces no tenía más formación que la educación básica (Ninh, 2002).

La figura de artista independiente se empieza a forjar en Vietnam tras la apertura en 1913 de la Escuela de Arte de Gia Dinh, en Saigón, primer centro educativo de esta naturaleza. La figura de artista como profesional individual quedó reforzada tras la inauguración de la Escuela de Bellas Artes de Indochina, proyecto colonial que operó en Hanoi de 1925 a 1945. Como cabe esperar, esta nueva noción de ocupación profesional

\footnotetext{
${ }^{4}$ A la largo de este artículo se usa la denominación Vietnam para referirse al territorio en su configuración actual, si bien ha tenido diversos nombres a lo largo de la historia. Formó parte de la colonia francesa de Indochina, desde finales del siglo XIX hasta su independización a mediados del siglo XX, cuando quedó dividido en Vietnam del Norte y Vietnam del Sur, países reunificados en 1976.

${ }^{5}$ El autor y editor Pham Quyhn sentía devoción por este poema épico, proclamando durante el periodo colonial que "mientras perdure La historia de Kieu, nuestra lengua perdurará; mientras perdure nuestra lengua, nuestro país perdurará"; traducción propia de "as long as The Tale of Kieu lasts, our language will last; as long as our language lasts, our country will last" (Vinh, 1995: 206).
} 
venía acompañada de la nomenclatura correspondiente. En el imaginario y en el idioma de Vietnam, nace la profesión de họa sĩ, término que se traduce propiamente como pintor/a o escultor/a aunque es frecuente que se use genéricamente para decir artista. En la sociedad vietnamita de ese periodo, họa sĩ designaba a quien creaba pinturas o esculturas para admirar en sí mismas, en contraste con thơ vẽ, término que se refiere a la persona que pinta, talla o esculpe ejerciendo un oficio artesanal, por ejemplo, para crear los intrincados templos de madera tallada y lacada.

En época colonial entraron en uso abundantes sino-neologismos, es decir, palabras acuñadas en China y Japón para designar los novedosos conceptos que emergieron por el contacto con occidente ${ }^{6}$. Palabras derivadas del francés y del ruso también dieron a la lengua vietnamita miles de vocablos nuevos para describir nociones asociadas con la modernidad (Chotpradit et al, 2018). A partir de la década de 1920, se constata la entrada al vietnamita de términos relacionados con eso que llamamos arte, y que difícilmente se puede definir con concreción, como bien sabemos por la cantidad de debates que existen al respecto, y porque la noción misma de arte no es unívoca ni en el tiempo, ni en distintas sociedades. No obstante, la palabra que en no pocos países de Europa es art o arte - significante de la concepción de arte que se tenía en Europa a principios del siglo XXen vietnamita toma dos formas: mỹ thuật o nghệ thuật (Chotpradit et al, 2018). El sufijo thuật conlleva una acepción de método o tecnología, que en la traducción nghệ thuật viene reforzada con el componente $n g h e ̣$, que significa técnica o destreza. Ahí donde $n g h e ̣$ thuật se refiere al arte en un sentido genérico, mỹ thuật corresponde al sintagma bellas artes, por incluir el prefijo $m \tilde{y}$, fino.

\subsection{Traducir el arte de Francia}

Durante la colonización francesa de Indochina, un público deseoso de conocer más sobre las novedades culturales y visuales que se estaban desarrollando dentro y fuera de Vietnam, asistía a las cada vez más frecuentes exposiciones de arte. La difusión de todo tipo de ilustraciones y caricaturas en periódicos contribuyó a la expansión del disfrute de la cultura visual. Las exposiciones de arte se realizaban mayoritariamente en Hanoi, aunque naturalmente las publicaciones alcanzaban una audiencia mucho más amplia. A partir de los años treinta, los semanarios Phong Hóa y Ngày Nay, maquetados con tipografía en quoc ngu, eran leídos por decenas de miles de lectores, incluso llegando hasta Laos y Camboya. Estas revistas publicaban ilustraciones hechas por artistas formados en la Escuela de Bellas Artes de Indochina (Scott, 2009). Entre estas nuevas actividades creadoras, reviste especial importancia la aparición de la obra firmada con nombre de artista, costumbre individualista desconocida hasta ese momento en la cultura local, resalta Huynh (2005). Cabe señalar que la pintura había sido escasamente

\footnotetext{
${ }^{6}$ Términos acuñados en el japonés de la época Meiji, como libertad (jiyu), gobierno republicano (kyowa), derechos (kenri), sociedad (shakai), evolución (shinka) o economía (keizaigazu), atravesaron China antes de alcanzar Vietnam, acarreando algunas distorsiones en el proceso (Vinh 1995: 206).
} 
practicada en la época pre-colonial de Vietnam, lo que hace más llamativo el rápido éxito de esta práctica artística en las escuelas de arte. Las clases de pintura eran mucho más populares que las de escultura.

Otro momento de gran transformación artística llega en las postrimerías del siglo XX, una vez superado lo peor de la posguerra. Llegados los últimos años noventa, se observa la tendencia a prescindir del vocablo hoa sĩ, (persona que pinta o esculpe) para referirse a la generación joven de artistas, quienes se identifican con los términos nghệ sĩ, artista, o nghệ sĩ thi giác, artistas visuales. Tras la aparición de obras en formatos como la performance, la instalación y el vídeo, se empieza a hablar de nghệ thuật đuoong đại, de arte contemporáneo, encontrando ocasionalmente la frase mỹ thuật đương đại, algo así como bellas artes contemporáneas. La popularización de nghệ thuật obedece a que connota una práctica artística más variada que el repertorio tradicional asociado con la concepción de bellas artes, mỹ thuạt, explican Phoebe Scott y Nguyen Nhu Huy (Chotpradit et al, 2018). Por el contrario, según el mismo estudio, la opinión del crítico de arte Nguyễn Quân es que el rechazo del modelo decimonónico de bellas artes implícito en el término mỹ thuật se expresa diferenciando los adjetivos contemporáneo (đương đại) o moderno (hiện đại). No deja de ser confuso que tanto đuơng como hiện significan actualmente, presente. Seguidos de đại (edad, época), ambas palabras describen el mismo fenómeno, algo nuevo, comenta la periodista cultural Dao Mai Trang (2016), quien muestra cierta exasperación por la cantidad de acepciones y definiciones de arte contemporáneo y de artista que se usan en Vietnam.

Aún mayores debieron ser la confusión y el desbordamiento a principios del siglo XX, cuando estaban difundiéndose tanto el alfabeto romanizado, como las nuevas palabras de diversos orígenes, así como algunos conceptos localmente novedosos. Todo ello acompañado por grandes cambios administrativos, jerárquicos y educativos. Entre los cambios jerárquicos está la irrupción de la profesión de artista, que entra en existencia en su modelo europeo decimonónico, es decir, como un trabajo de carácter singular y no gremial. No quiere esto decir que en Vietnam se haya efectuado una importación directa de la glorificada mística del genio, sino que el propio idioma vietnamita juega un papel en la transmogrificación de la labor creadora. Históricamente, el idealizado orden social confuciano reconocía jerarquías que iban de los intelectuales (sĩ), pasando por el campesinado (nông), hasta trabajadores de artesanado (công) y en último lugar, situando a las personas dedicadas al comercio (thuong). Por ello es revelador que las palabras acuñadas para decir artista, nghệ sĩ y hoa sĩ, emplean el sufijo sĩ, tradicionalmente asociado con la clase erudita (Chotpradit et al, 2018).

Nada parece indicar que el alto estatus de la profesión de artista se haya puesto en cuestión a posteriori, ni durante la resistencia anticolonial ni tras la independencia del país a mediados del siglo XX, si bien en las décadas siguientes hubo debates sobre el perjuicio que suponían para las tradiciones y la cultura local algunos conceptos modernos importados del extranjero. Es más, una vez que la profesión de artista estaba bien implantada, fue un arma ideológica contra las fuerzas invasoras, ya que fueron las 
personas que se identificaban con la nueva actividad pintora quienes lideraron nuevas formulaciones para pensar las bellas artes (Nualart, 2017). La lucha contra la ocupación extranjera fue sin duda una lucha intelectual, con vivo debate sobre el choque entre perspectivas supuestamente autóctonas y foráneas, tal y como sucede con el arte.

Por el contrario, algunos inventos importados, como los triciclo-taxis, se convirtieron en parte del paisaje urbano de Vietnam sin que su origen francés causase furor alguno. La asimilación es visible en la misma palabra que les da nombre. El idioma galo ha dejado su impronta en el vietnamita aportando vocablos de clara afinidad acústica como xích lô (cyclo[-pousse], triciclo).

\subsection{Dibujar en vietnamita. El arte de Truong Tan}

Retomando la cuestión de la representación visual de las palabras, si el sonido de vocablos franceses se adapta a la forma de escritura vietnamita (en quoc ngu), cabe preguntarse si la forma francesa de representar imágenes - que no palabras - se adaptó al vietnamita. Es decir, ¿se puede dibujar en francés? La pregunta la hace Truong Tan (n. 1963), un artista que en la década de 1990 fue profesor en la Universidad de Bellas Artes de Hanoi, institución heredera de la antigua Escuela de Bellas Artes de Indochina, y seguidora de ese modelo educativo francés enfocado en el manejo de medios, técnicas y estilos artísticos europeos. El dibujo del natural, frente a modelos en pose, fue parte imprescindible de esa formación.

Un dibujo del artista Truong Tan presenta la imagen de un desnudo masculino sedente bajo el rótulo Báo Tón Mỹ Thuật, juego de palabras que viene a reivindicar una defensa del arte bello o de las bellas artes, y que se tradujo al inglés como Defend Beautiful Art, perdiendo así la referencia a las bellas artes (en inglés, Fine Arts). El bosquejo del musculado varón, en torsión cual discóbolo de Mirón, magnifica la carga homoerótica de los cuerpos lampiños de la estatuaria clásica. Pero no es en esa dirección donde se dirige la intención de Truong Tan, primer artista vietnamita en salir del armario (Nualart, 2016).

En su búsqueda de respuestas a la pregunta ¿se puede dibujar en francés?, Truong Tan recurre al dibujo, la escritura, el cuerpo y el espacio público. El artista estampa una camiseta con su dibujo del modelo masculino sedente. Bajo la imagen se lee otra frase más: E con de Bo-za (1925-1997). Es una transcripción fonética en vietnamita del nombre francés École des Beaux-Arts de l'Indochine, o EBAI, la famosa institución colonial promotora de los paradigmas occidentales que desestabilizaron las nociones precoloniales sobre la actividad creadora. Los estudiantes que se habían formado en la institución antes de su cierre en 1945 están ya canonizados como los más importantes artistas modernos de Vietnam, no obstante la desfasada enseñanza ofrecida en la renombrada escuela, cuyo currículo enfatizaba temas y técnicas academicistas, mientras que ignoraba las aportaciones europeas del Dadá, el cubismo o el surrealismo, señala la curadora Iola Lenzi (2016). 
La EBAI como institución nació en 1925, como indica la esquela escrita por el artista en la camiseta, pero los veinte años de funcionamiento de la escuela han tenido una repercusión tan inmensa y larga en la historia del arte vietnamita, que hubo que esperar a que Truong Tan decidiese que había llegado la hora de clausurarla, para percatarse de había dejado de operar en 1945. Truong Tan comprendió la necesidad de una reflexión crítica sobre el legado de la EBAI, y el momento oportuno fue el año $1997^{7}$, transcurridos diez años desde el doi $m o i^{8}$. Estas importantes reformas políticas en Vietnam facilitaron entre otras cosas la apertura de las primeras galerías de arte privadas. Apenas hacía dos años que se había realizado la acción que la especialista Nora Taylor (2007) considera puede ser la primera obra de arte de performance en el país, de la que parece ser que solo hay testimonios orales ${ }^{9}$. Por otro lado, un libro vietnamita escrito por Bui y Pham (2012) atribuye a Truong Tan las primeras performances del país.

Se documentaron poco o nada las performances de ese periodo formativo, pero sí se tomó una fotografía de Truong Tan realizando una acción que pasó desapercibida entre el trajín cotidiano de un mercado [figura 1]. Vestido con su camiseta, el artista aparece sentado sobre un taburete del mismo modo que la figura impresa en la prenda. Permanece como en pose, sin dibujar, dejando que el dibujo sobre su torso hable por él. Esta performance, que apenas ha tenido el eco que seguramente merece, nos dirige a una de las más pertinentes preguntas sobre el concepto de arte: el espacio donde se toma contacto con el hecho artístico, en este caso, un mercado.

\footnotetext{
7 Tanto esta performance de Truong Tan, como la obra más destacada de Amanda Heng comentada más adelante, están fechadas en 1997, el año de la crisis financiera asiática. Un año antes, en 1996, se había inaugurado en Singapur el museo de arte SAM (Singapore Art Museum). En estos años circula entre los profesionales en el arte del Sudeste Asiático mucho debate sobre la historia del arte regional, como se vio en la introducción.

${ }^{8}$ En 1986 tuvo lugar el doi moi, la renovación política y económica que abrió Vietnam al turismo, al comercio internacional y permitió la creación de empresas privadas.

${ }^{9}$ En relación con los posibles orígenes, que rara vez son únicos, de la performance en Vietnam, hay documentación visual de una performance realizada en la Facultad de Bellas Artes de Hanoi, aunque la artista no es vietnamita, sino de Singapur (Radulovic, 2009: 88-89). Amanda Heng, a quien más adelante se dedica buena parte de este trabajo, fue invitada a Hanoi por Veronika Radulovic quien ejercía de profesora de arte en la Universidad de Hanoi en ese momento. Radulovic forjó amistad con Truong Tan que había sido estudiante y luego profesor de esta universidad.
} 


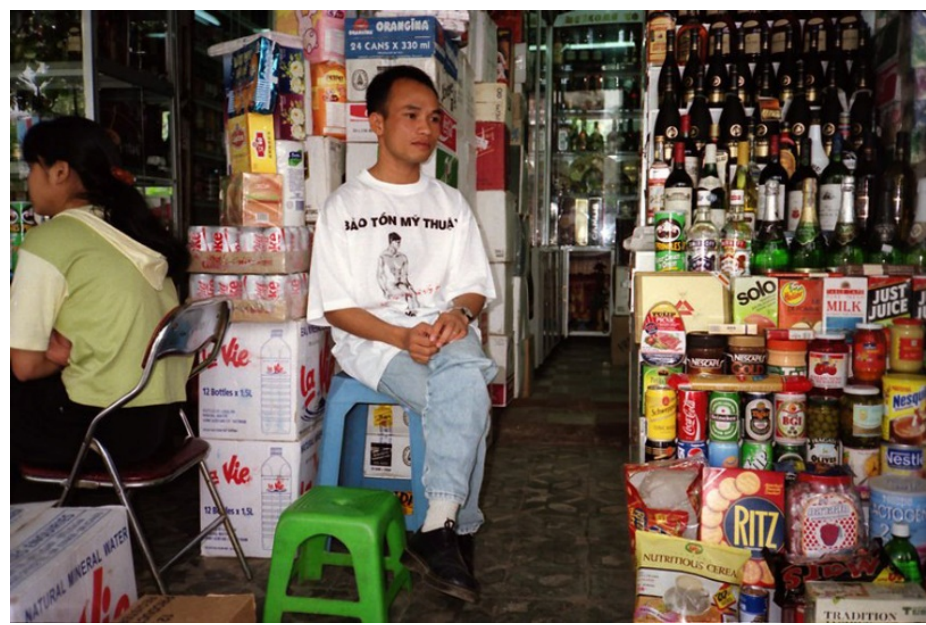

Figura 1: Performance del artista Truong Tan en el mercado Hang Da de Hanoi, Vietnam, 1997. Fotografía de Veronika Radulovic, usada con permiso.

La fotografía de la colección de Veronika Radulovic [figura 1] muestra a su amigo el artista sentado sobre un taburete en un puesto del mercado (Cho) Hang Da de Hanoi. Ese mercado fue el primero que en Vietnam donde se comercializaron deseables productos occidentales de importación, dato histórico de evidente relación con la crítica que hace Truong Tan. Un análisis de semiótica estética permite ver en esta pieza cómo la historia de una nación se ramifica cual árbol genealógico.

Descomponiendo los diferentes significantes, encontramos impresa en la camiseta la imagen de un dibujo del natural, que representa a un modelo desnudo. Por ser típica de una clase de arte academicista, hace referencia a la educación artística de los albores del siglo XX. También a ese periodo apela la invocación a defender la belleza en el arte escrita sobre la camiseta, cuyo significado es más complejo: la entrada lingüística y conceptual en Vietnam de la noción occidental de bellas artes.

Seguidamente, la fecha de defunción de la herencia colonial inventada por el artista, 1997, queda registrada en el slogan que viste. La fecha escrita en la camiseta de Truong Tan simbólicamente envía a la guillotina la obsolescencia de las bellas artes, mientras luce su poema visual — si puede considerarse así la imagen de su camiseta - y practica una nueva manifestación artística, la performance. Lo más destacado, es sin duda la inteligencia verbal encarnada en esta creación. Qué golpe visual es $E$ con de Bo-za, traducción fonética del francés al vietnamita, para afirmar resueltamente que la escritura y la lectura propia aportan significados que ningún préstamo literario o neologismo puede dar.

No es probable que en 1997 las autoridades hubiesen concedido permiso a Truong Tan para realizar su performance, ya que la acción era, según Radulovic (2012), una de las características provocaciones de este artista rebelde. Parte de la transcendencia del arte de performance en Vietnam fue precisamente que su carácter efímero permitía ahorrarse las gestiones de pedir a la policía cultural las licencias necesarias para inaugurar exposiciones o eventos artísticos, siempre y cuando las representaciones ocurriesen en 
locales privados o en lugares insospechados, sin aparatosidad alguna (Taylor, 2007). Y cómo no iba a pasar desapercibida la actitud casual de un joven en camiseta y vaqueros observando a la gente pasar por el mercado. Al fin y al cabo, quién iba a identificar al enfant terrible del arte vietnamita en ese lugar, si solamente escasas personas vinculadas con el mundo del arte le hubiesen reconocido por la calle. Menos aún hubiesen entendido las inscripciones de su camiseta.

No obstante, el arte de performance no tuvo una acogida desenfrenada, ni por parte de artistas ni de expertos en arte. En 2005 varios académicos del Instituto de Cultura y Arte de Vietnam declararon que las performances y las instalaciones no eran ni arte, ni vietnamitas, alegando que eran feas y estaban alejadas de las tradiciones nacionales. Según estos críticos, eran los artistas que copiaban de occidente quienes hacían este tipo de creaciones, y por lo tanto no podían ser "verdaderos artistas vietnamitas" (Taylor, 2007: 108). Paradójico razonamiento, aquejado simultáneamente de nacionalismo vietnamita y de defensa del academicismo europeo. Este ataque oficial a las artes nuevas pasa por alto que la invasión colonial contribuyó a modelar las concepciones vietnamitas sobre el hecho estético ${ }^{10}$.

El breve tiempo de la sentada de Truong Tan vestido con el lema E con de Bo-za (1925-1997) en el mercado de Hanoi condensa los choques artísticos que se sucedieron en Vietnam en el siglo XX. Siendo el objetivo de la performance el de manifestar oposición a la estructura del conocimiento artístico hegemónico, consideramos que la traducción fonética creada por el artista, en su soporte multidisciplinar, se puede entender como una traducción heteroglósica, cuyos diversos mensajes arremeten contra el mundo del arte de su entorno tanto en la época colonial como la del momento.

Por una parte, el artista visibiliza la persistencia del legado de la formación artística colonial, dominante desde 1925 hasta 1997. Por otro lado, subraya la interrelación del arte con el comercio, al elegir expresarse, con la escritura por bandera, en un mercado. La ironía en este sentido es clara, comenta Radulovic (2012) conocedora del giro comercial del arte vietnamita que hasta poco antes estaba subvencionado por el estado. Efectivamente, durante las décadas de guerras, el gobierno comunista de Vietnam del Norte remuneraba a sus artistas como trabajadores de arte, a través de la Asociación Nacional de Artistas creada en 1957 (Fan, 2009) ${ }^{11}$.

Las camisetas como objetos artísticos comercializables llevan más allá si cabe la crítica colonial e institucional de la performance. Tras realizar su performance, Truong Tan creó una edición limitada de la camiseta que había diseñado, añadiendo otra frase al

\footnotetext{
${ }^{10}$ Recordemos que cuando llegó a Vietnam la acepción de bellas artes, en Europa el cine se acababa de proponer como el séptimo arte. Parecer quedar tan lejos ese momento que pone de manifiesto lo jóvenes que son las bellas artes todavía, en comparación con las artes de las nueve musas del mundo clásico, por su parte más próximas a oriente que a París. Haciendo honor a la evolución creativa, las concepciones de arte y sus formas de categorización nunca han permanecido fijas durante demasiado tiempo en ningún lugar.

${ }^{11}$ Es cierto que, hasta la reunificación de ambos territorios en 1976, la situación fue diferente en Vietnam del Sur, donde las circunstancias posibilitaban, dentro de los límites de la situación de guerra, el comercio privado de obras de arte.
} 
diseño, texto donde aparece una nueva capa de significado. Sobre cada camiseta escribió a mano la oración $O$ day, chung toi van con ve tieng phap (Aquí todavía dibujamos en francés), adaptando el slogan que en 1997 publicitaba la Cumbre Francófona en Hanoi: Aquí todavía se habla francés (Radulovic, 2012). El mensaje avisa del posible retroceso que supondría mantener activa la colonización lingüística, o en su caso la educación colonial en bellas artes. La influencia en el arte de las transferencias de conocimiento internacionales es continuada y global, en todas direcciones, y sin duda siempre ha sido así, aunque falten estudios que descubran qué transferencias de conocimientos periféricos han llegado al centro.

Para el historiador Alexander Woodside, Vietnam es y ha sido una de las civilizaciones más intensamente literarias del mundo (Ninh, 2002). Ello reviste de importancia el juego de reconocer grafías y significados que el artista Truong Tan incorpora a su práctica. Ha integrado palabras tanto pintándolas en sus cuadros de óleo o de laca, en sus piezas de cerámica o en sus performances, mezclando distintas lenguas para contraponer significados divergentes. Así puede poner palabras como la inglesa fuck, cuya malsonancia en contextos angloparlantes es ajena a oídos no nativos, y lo suficientemente extraña para no ser sexualmente provocadora. Otro recurso de Truong Tan es elegir títulos multilingües, como es el caso de Xin Loi-Excuse Me-Excusez Moi (HKW, 1999).

El carácter subversivo del lenguaje brilla en la frase E con de Bo-za (1925-1997), ya que hace referencia a la noción de lugar (además del tiempo, en las fechas). El lugar que nombra, la escuela colonial EBAI, es inseparable en la historia del arte de Vietnam, pero el lugar de la performance realizada por el artista, el mercado de prestigiosos bienes de consumo implica un cuestionamiento de los espacios donde se presenta y se comercializa el arte. ¿Se puede dibujar en múltiples lenguas en un mercado? Sin duda, parece responder el artista, ahí es donde tiene que hacerse el arte.

\section{SINGAPUR: EL ROBO DE LA LENGUA MATERNA}

La artista de Singapur Amanda Heng también empezó a mediados de la década de 1990 a usar el espacio público como espacio de creación, y desde entonces es apreciada por reflejar en sus obras temas de identidad comunitaria y memoria histórica. Heng fue una pionera de la performance en la calle. La artista, partiendo de su experiencia como mujer de ascendencia china en un Singapur muy occidentalizado, es autora de obras que dan espacio a múltiples voces de diversas culturas, entornos, disciplinas y perspectivas.

\subsection{Políticas culturales para construir identidad social}

La antigua colonia británica de Singapur se convirtió en una ciudad-estado independiente en 1965. Las dos décadas que siguieron al nacimiento de Singapur como país fueron de éxito singular en lo político y lo económico. Cierto es que la pequeña 
nación multicultural inició su independencia con pocos recursos y rodeada de vecinos poderosos, situación que llevó al partido gobernante (People's Action Party, PAP) a idear unas "políticas de supervivencia" consistentes en imponer una autodisciplina a la ciudadanía, con medidas dirigidas al control y a la movilización social, así como a atraer inversión extranjera para empujar el crecimiento económico (Steinberg, 1987: 415). Escasa corrupción, consultas ciudadanas y la provisión de una gran variedad de servicios sociales de calidad permitieron al gobierno ejercer cierto autoritarismo, tolerado porque la calidad de vida de Singapur rivalizaba solo con la de Japón.

Cuando Amanda Heng (n. 1951) era niña e iba a la escuela, el sistema educativo nacional fomentaba el aprendizaje del inglés y el mandarín, y premiaba el alto rendimiento, preparando así a la sociedad para entrar en un competitivo mercado de trabajo con fuerte demanda tecnológica. Las incertezas sobre la identidad social singapurense emergieron más adelante, cuando durante década de 1980 se quiso dar al confucianismo un papel más importante dentro de la cultura nacional, remedio peor que la enfermedad, que no resolvió las cuestiones identitarias (Steinberg, 1987). Los efectos secundarios de las políticas de fomento del inglés como lengua franca para Singapur han abierto brechas en el uso de la herramienta más directa de expresión personal, el lenguaje. En palabras de la artista:

Lo peor que se puede hacerle a una persona, es negarles su idioma y su lengua materna, porque entretejido en el lenguaje están su cultura, sus creencias y sus valores; el lenguaje es el alma de la persona, algo por lo que se siente orgulloso. Este país ninguneó los dialectos y no comprendió su valor. Las cosas se cambiaron de la noche a la mañana y de repente, madre y las generaciones mayores no debían usar su propio dialecto para hablar con sus hijos. Por supuesto la gente continúa usando dialectos, pero había un estigma. Te miraban mal... Tu propia cultura quedaba reducida pero todo lo que venía de Occidente era bueno. La gente joven evitaba los dialectos. Afectó incluso la forma en la que comemos. ¿Cómo puedes convertirte en una persona centrada cuando la lengua de tus padres de repente se convierte en algo casi ilegal? (Black, 2015) ${ }^{12}$.

\subsection{Abrazar la lengua materna. El arte de Amanda Heng}

Tras muchos años trabajando para la agencia de tributación, Amanda Heng dio un vuelco a su trayectoria profesional, y se reinventó como artista a finales de la década de 1980. Heng apareció en la escena en un momento histórico de transformación artística en Singapur, cuando la performance, la instalación y otros formatos multidisciplinares de

\footnotetext{
12 Traducción propia de: "The worst thing to do to a person, is to deny them their language and mother tongue, because embedded in the language are their cultures, beliefs and values; language is a person's soul; something they are proud of. This country belittled dialects and failed to understand its values. Things were changed overnight and all of a sudden, mother and the older generation were not supposed to use their own dialect to talk to their children. Of course people continue to use dialect but there was a stigma. You were looked down upon... Your own culture was downplayed and everything from the west was good. Young people tried to avoid dialects. Even the way we eat was affected. How can you become a centered person when the language of your parents suddenly becomes something almost illegal?" (Black, 2015).
} 
arte contemporáneo irrumpieron en espacios públicos y comunitarios (Rawlings, 2010). En 1988, Amanda Heng colaboró en la fundación del colectivo The Artists Village, un hito en los primeros pasos del arte contemporáneo singapurense. El año que Truong Tan realizó su performance en el mercado de Hanoi, Amada Heng consiguió un taller en Telok Kurau, complejo de estudios para artistas apoyado por el Consejo Nacional de Arte de Singapur. Antes de eso, Amanda Heng ya había incorporado ideas feministas a su arte, pero es cuando tiene un estudio de arte cuando acoge mensualmente a otras artistas para dar charlas, intercambiar ideas y organizar un archivo de artistas mujeres (Rawlings, 2010). En 1999, Heng lideró el nacimiento del colectivo Women In The Arts Singapore (WITAS). El propósito de Heng como artista es el de incitar al público a reflexionar sobre el contexto multicultural de Singapur, en particular sobre cuestiones sociales como los cambios culturales o la identidad de género y de grupo. La preferencia de Heng de trabajar en la calle, proponiendo acciones que llegan a la gente común, no facilitó su presencia en exposiciones. Su primera exposición individual, Speak with Me, Walk with Me, tuvo lugar en 2011 en el Museo de Arte de Singapur (SAM), mostrando dos décadas de su práctica artística. Performances públicas de Amanda Heng se han efectuado en la Bienal de Singapur de $2019^{13}$.

De la producción de esta artista tomamos un par de obras centradas en políticas lingüísticas y el uso controlado de las lenguas y de la escritura. La performance $S / H E$ (1994), en primer lugar, exploraba cómo algunos conflictos culturales, como el choque entre la cultura oriental y la occidental, afectan la construcción de identidad. Confrontando el sexismo inherente al imperialismo oriental y al occidental, Amanda Heng cuestionaba lo que significa ser mujer en el contexto político y cultural de su tierra natal, Singapur. Vestida de negro para la representación de $S / H E$, la artista se maquillaba como una hua dan, personaje femenino encarnado por actores varones en la ópera tradicional china. Durante la acción, Heng pintaba sobre su rostro símbolos derivados de alfabetos chinos, al tiempo que intentaba pronunciarlos frente a un espejo, desbrozando el lenguaje hasta dejarlo en sus fonemas y marcas más simples. $S / H E$ es una performance que Amanda Heng repitió varias veces en 1995 y 1996, enriqueciéndola con nuevos significados cada vez, por ejemplo, usando masa de pan para borrar de su cara las marcas pintadas mientras recitaba las tres obediencias exigidas a la mujer en el pensamiento confuciano: obedecer al padre, obedecer al marido, obedecer al hijo. Arrojando con fuerza la masa de pan, la artista manifestaba gestualmente su rechazo a los roles tradicionales de la mujer como sirvienta de los hombres.

La historiadora del arte y curadora Adele Tan (2015) encuentra fascinante la conjunción de cuerpo y de lenguaje en las obras de Heng de principios de los noventa. El encuentro entre palabra y carne en estas tempranas performances es incisivo por el modo en el que consigue relegar el lenguaje que, en la práctica, ha causado la anulación de la existencia social de las mujeres, explica Tan (2011). Las reivindicaciones feministas en

${ }^{13}$ Véase: https://www.singaporebiennale.org/art/amanda-heng [06/04/2021]. 
la obra de Amanda Heng son transcendentes en el discurso artístico global de los años noventa.

Este trabajo persigue ahondar en los usos culturales del lenguaje, y para ello se analiza la obra Another Woman (1996-1997), que contiene un autorretrato de Amanda Heng, aunque no está sola. Habitualmente, Heng trabaja con el público general y con más artistas, pero en esta ocasión, y no por primera vez, la artista colabora con su madre. En esta instalación de fotografías y objetos (una jaula de pájaro), el protagonismo se lo llevan las fotografías, que atrapan la mirada ${ }^{14}$. Las fotografías de un abrazo en primer plano transmiten el afecto entre la artista y su progenitora, si bien están tomadas para poner en evidencia el desplazamiento cultural que ha nacido entre madre e hija. Otra fotografía de la serie es un primer plano de las manos de las dos mujeres, una con la palma abierta, sobre la que se ha escrito la palabra Love. Conocer a su madre es un proceso necesario para descubrir su propia identidad y valores, explicó Amanda Heng en una entrevista, añadiendo que la memoria y la historia se deben repensar críticamente, especialmente en la formación de nuevas creencias y valores vitales (Black, 2015).

El cálido abrazo entre dos mujeres que aparece en Another Woman acerca a dos generaciones cuya separación es mayor que una natural diferencia de edad. Esto se debe a que en un momento de fuerte desarrollismo del Sudeste asiático, madre e hija sufrieron los efectos de una separación comunicativa, aun conviviendo bajo el mismo techo. Fue una consecuencia insospechada de las políticas educativas impuestas en Singapur, comentadas anteriormente. Las estrategias de creación de nación (nation-building) puestas en marcha en la isla recién independizada desincentivaron el uso de dialectos como el teochew, la lengua nativa de la madre de Amanda Heng. Koh Siam Tian, madre de la artista y de ocho hijos más, nació en una zona rural de China en 1925, pero fue parte de la gran diáspora del pueblo teochew (Wubin 2016). La comunidad teochew es relativamente grande entre la población multiétnica de Singapur, lo que no evitó que las políticas gubernamentales empezaran en la década de 1960 a privilegiar la enseñanza y el uso del inglés sobre el mandarín y los dialectos. Se cerraron las escuelas chinas, malayas e indias, o bien pasaron a enseñar en inglés. La radio y las noticias televisivas tenían prohibido emitir en dialectos, dejando a las generaciones más mayores sin entretenimiento e información sobre la actualidad. En este clima tiene su origen la fisura comunicativa en la familia de Amanda Heng. La artista fue la única educada en mandarín, sus hermanos estudiaron en inglés, mientras que la madre solo habla con fluidez el dialecto teochew y aunque Amanda Heng es teochew, no habla bien esa lengua. No es sorprendente, pues, que a Amanda Heng le había resultado difícil explicar a su madre su decisión de dejar su puesto de funcionaria para hacerse artista, o hacerle entender su práctica artística (Wubin, 2016).

\footnotetext{
${ }^{14} \mathrm{La}$ fotografías de Another Woman de la colección del Singapore Art Museum se pueden ver en: https://www.singaporeartmuseum.sg/about/blog/6-things-to-know-about-amanda-heng-and-her-workevery-step-counts [06/04/2021].
} 


\subsection{Palabras para destruir y reconstruir la identidad familiar}

En lo concerniente a la materia misma del lenguaje que emplea la artista, el inglés, destaca de esta obra el vocabulario elegido para titularla. Las palabras Another Woman parecen recalcar la separación que hay entre madre e hija, aunque en realidad se refieren a la decreciente identidad social de la madre, por el creciente desprestigio de las lenguas no hegemónicas que las políticas educativas estaban generando.

Ambas abrazantes son otra. Si la palabra another en el título evidencia la otredad, la elección del sustantivo woman, mujer, descriptor genérico de persona de sexo femenino, deja fuera del campo semántico cualquier referencia a vínculos familiares. En una relación donde cabría hablar de kinship, de relaciones de sangre, de madres e hijas, allegadas, parientes - en definitiva, de lazos familiares - las palabras que dan nombre a la obra proyectan una individualidad anónima y desafectada. La frialdad del título apela tanto en su medio como en su mensaje al poder del idioma como barrera, como instrumento para marcar distancia. Todo lo contrario sucede con el abrazo en color de la fotografía, que nos devuelve al calor humano.

Pasados veinte años desde esa obra, Amanda Heng retomó su exploración del conocimiento propio y de su madre por medio de abrazos, recreando el gesto en una segunda parte, creando la fotografía titulada Twenty Years Later (2015). Nuevamente vemos el rostro de la madre con los ojos cerrados, abrazada por la artista que nos da la espalda, ahora ella también con el pelo blanco. Como espectadores, estamos muy cerca de este abrazo que llena la composición. La fotografía de Twenty Years Later está tomada desde un poco más cerca incluso que Another Woman, donde ya solo veíamos la parte superior de los cuerpos. Las dos mujeres también se han acercado entre ellas, han cerrado el pequeño espacio que las separaba en la imagen primera. Es quizás una señal de que han progresado en conocerse más a fondo y han superado el obstáculo de las políticas culturales estatales que las desunió.

El desplazamiento generacional que marca desde siempre el compás de la humanidad es otra aportación que Another Woman consigue transmitir. La madre de Heng, procedente del campo, sobrevivió a la guerra, se casó y siguió la costumbre china de cuidar a los padres de su marido, a sus hermanos, y a sus propios nueve hijos. Todo esto en un Singapur aun pobre. La población predominantemente china, conocedora del dicho maoísta las mujeres sostienen la mitad del cielo, sostenía que el esfuerzo de construcción de la nación era de todas y todos, una cuestión pragmática más que emocional (Kokuritsu Shin Bijutsukan, 2017: 118). Décadas después, en el momento de creación de Another Woman, en Singapur se tensaron las relaciones familiares debido a la posición cambiante de las mujeres, causando recelo en entornos jerárquicamente ordenados por las costumbres confucianas ${ }^{15}$.

\footnotetext{
${ }^{15}$ A pesar de ciertas conquistas en las dinámicas familiares respecto a generaciones anteriores, las mujeres sufrieron más que los hombres la crisis financiera de 1997, dando a Amanda Heng motivo para caminar en
} 
La experiencia vivida por Amanda Heng y su madre evidencia la enorme dificultad de diseñar políticas que cumplan su función — en el caso de Singapur el de fomentar una lengua común a su diversa ciudadanía - sin que los fracasos sean exacerbados por cambios nefastos en el conocimiento social. Las formas de pensar de una sociedad son permeables a la acción colectiva, e inciden a su vez sobre ella. Si Amanda Heng vivió un problema de entendimiento con su madre, la dificultad no quedó en el ámbito doméstico, sino que se vivía en el espacio público, donde como se ha visto, el uso del mandarín y de dialectos estaba muy mal visto. El estímulo al entendimiento quedó polarizado, dejando atrás a los más débiles que por edad, obligaciones o limitada escolarización tenían menos facilidad y oportunidades para aprender un nuevo idioma.

A través de sus circunstancias personales, Amanda Heng aborda el desplazamiento en el tejido social que una política cultural puede producir a nivel nacional y a nivel familiar. Como contrapartida, las fotografías de abrazos maternos fomentan un nivel universal de lectura, difusor del sentimiento ineludible de que materialmente, no hay persona que nazca careciendo de ancestros.

\section{DISCURSO, LENGUAJE Y COLONIZACIÓN}

Afincado en Singapur, el crítico de origen malayo Lee Weng Choy (Weng 2009) vio como en la década de 1990 profesionales del arte internacionales reconocieron que el futuro de la historia y la crítica de arte ya no pertenecía a occidente, sino que era global. Los cambios en estas disciplinas los atribuye en parte al auge del arte contemporáneo de Asia. Lee Weng Choy, al igual que Nora Taylor (2012), están entre los observadores del arte asiático que en la década del 2000 afirmaban que el discurso postcolonial se había convertido en un sistema epistemológico dominante, debido a que el uso rutinario desde los años noventa le había restado fuerza crítica. Es importante por ello valorar la crítica colonial presente en las obras comentadas anteriormente, realizadas antes de este giro en la argumentación crítica de corte postcolonial. Truong Tan y Amanda Heng están trabajando en un momento de mucho vigor intelectual en sus sendos países. La vitalidad está motivada en el caso de Vietnam por la boyante situación económica y social, y en el caso de Singapur, que ya era una potencia económica, por las nuevas dinámicas artísticas: la inauguración del Singapore Art Museum, el trabajo innovador de artistas como Tan Da $\mathrm{Wu}$, Lee Wen y Amanda Heng. Tanto en Truong Tan como en Heng, se percibe una reflexión histórica, por sus formas de analizar el periodo que viven.

La cuestión que principalmente se quiere poner en valor en este estudio es la singular atención que los artistas han prestando al lenguaje en sus obras. El lenguaje tiene innegables vínculos con la geografía. Vietnam es una comunidad etnolingüística en la que se hablan diversas lenguas étnicas, aunque domine el vietnamita, mientras que en gran

la calle y visibilizar el sexismo en el entorno laboral. Su cómica performance Let's Walk (1999) fue representada en otros países, como Suecia o España. Véase: https://sagg.info/event/revisiting-lets-walkamanda-heng/ [06/04/2021]. 
medida Singapur, poblada por personas de orígenes chinos y malayos, experimentó una expresa unificación lingüística en los años sesenta y setenta del siglo XX. Resulta fascinante que Truong Tan haya evocado en una performance la milenaria supervivencia del vietnamita como lengua vernácula a pesar de las invasiones foráneas, y que Amanda Heng haya paralelamente abordado lo territorial desde una situación completamente diferente. Singapur, un país joven y multicultural, con una ciudadanía donde la norma es ser inmigrante o descendiente de inmigrantes, vive sus afectos por medio de la diversidad idiomática. Cuando esta tambalea, zozobra la sensación de seguridad del individuo.

La tradición asiática ha querido que la continuidad artística no sea rompedora, ni que ponga en cuestión la tradición, relata Heng, motivo por el que la performance, precisamente, es relevante, si bien su reconocimiento como práctica desde la década de los ochenta refleja un cambio mundial (Morelli, 2019). En los noventa, cuando encontrar y calificar lo regional en el arte del Sudeste Asiático era el rompecabezas de los historiadores del arte (Sabapathy, 1996; Weng, 2009; Taylor, 2012), la tradición se deja de percibir como algo monolítico. La performance, en estas circunstancias, es liberadora. En entrevista, Amanda Heng admite que el sufrimiento no es fácil de expresar en palabras, con el lenguaje oral, siendo más adecuado el lenguaje corporal, expresarse con el cuerpo, con la performance (Morelli, 2019). En la interpretación de Adele Tan (2011: 52), las obras de Amanda Heng efectúan una "comunicación encarnada", dado que tanto la forma como la agencia que las obras dan al habla tienen la misma importancia que el contenido albergado en el texto de las mismas, que es una denuncia pública. Más allá de cómo el arte consigue ser un medio de expresión, lo central de Another Woman es que los desplazamientos lingüísticos generados por decisiones políticas no solo alteran el tejido social en el espacio público, sino también remueven la esfera privada.

\section{CONCLUSIONES}

Cuando Colón navegaba buscando las Indias, Antonio de Nebrija quiso imponer en España una gramaticalización de la lengua vernácula que, según Illich y Sanders (2019: 88), estaba pensada para ser un sistema de control del pensamiento del pueblo llano. Los peligros del control lingüístico de una sociedad los han puesto en evidencia, ejemplarmente, tanto Truong Tan como Amanda Heng, empleando recursos del lenguaje visual y escrito, así como de la comunicación no verbal y de las prácticas de la traducción, transcripción y deconstrucción fonética.

Seguramente no es casual que el momento en el que Truong Tan y Amanda Heng empiezan sus trayectorias creadoras coincide con la diseminación de nuevas prácticas artísticas abiertas que buscan conectar la creación artística y la acción social, así como suspender la jerarquía entre quien crea y quien mira lo creado. Efectivamente, es a partir de la década de 1990 cuando comienza el auge de estrategias participativas y democratizadoras en el mundo del arte. Este periodo en Vietnam estuvo marcado por el giro económico y la apertura política de Vietnam, mientras que Truong Tan y artistas de 
su generación abrieron paso a la performance, al tiempo que otros agentes creaban los primeros espacios de arte autogestionados y colectivos artísticos independientes, como ocurrió en Singapur, con Amanda Heng al frente.

El objetivo de unificar Singapur con políticas destinadas a facilitar la interacción entre ciudadanos procedentes de distintos entornos tuvo como irónica contrapartida una mayor dificultad de comprensión intrafamiliar. Para Amanda Heng esto fue una victoria pírrica, y solo a posteriori se comprendió cuántas víctimas dejó por el camino (Tan, 2011). En este sentido, la obra de Heng es relevante por mostrar desde dentro un problema habitual de la migración, como es la adaptación a una nueva cultura e idioma. Sus personales obras inducen al público a preguntarse sobre valores éticos, tradiciones y la responsabilidad social, en el marco de la identidad cultural y la construcción de identidad nacional. Como se ha visto, el efecto de la alienación materna queda señalado en el título de la pieza autobiográfica Another Woman, palabras que establecen un paradójico distanciamiento entre la madre e hija fundidas en un abrazo.

Escribiendo los fonemas $E$ con de Bo-za, en el conjunto de su performance en un mercado, el artista vietnamita Truong Tan transmite una crítica a la educación artística y colonial en relación con los espacios públicos de comercio transnacional. La interacción de semiosis entre dibujo, performance, lenguas y transcripción fonética han sumado fuerza a una obra extraordinaria por su capacidad de sintetizar las claves de la historia del arte moderno de Vietnam.

En la introducción, se planteó la propuesta de Legaspi-Ramirez (2018) de preguntar cómo significan las obras de arte. La respuesta se buscó en piezas cuyos mecanismos de significación parecen ejercicios semióticos. La oralidad, la historia lingüística, los usos y abusos de los lenguajes en espacios públicos y privados, y los vínculos entre idioma y afectos son los protagonistas de las creaciones visuales presentadas.

\section{REFERENCIAS BIBLIOGRÁFICAS}

Black, S. (2015). "Amanda Heng / Performance Art in Context: A Singaporean Perspective by Lee Wen", Blacksteps.tv. Disponible en línea: http://www.blacksteps.tv/amanda-heng-performance-art-in-context-asingaporean-perspective-by-lee-wen/ [27/05/2018].

Bui Nhu Huong \& Pham Thuong (2012). Vietnamese Contemporary Art 1990-2010. Hanoi: Knowledge Publishing House.

Chotpradit, T.; Jacobo, J. P.; Legaspi-Ramirez, E.; Nelson, R.; Nguyen N. H.; Polmuk, C.; Tun, S. L.; Scott, P.; Soon, S. \& Supangkat, J. (2018). 'Terminologies of 'Modern' and 'Contemporary' 'Art' in Southeast Asia's Vernacular Languages: Indonesian, Javanese, Khmer, Lao, Malay, Myanmar/Burmese, Tagalog/Filipino, Thai and Vietnamese". Southeast of Now:

Directions in Contemporary and Modern Art in Asia 2.2, 65-195. 
Dao Mai Trang (2016). Art \& Talent. A foreground on the $8 X$ contemporary artists generation of Vietnam. Hanoi: Đào Mai Trang.

FAN, J. (2009). "Social Realism in Vietnamese Art". En Essays on modern and contemporary Vietnamese Art. S. Lee \& Nguyen Nhu Huy (eds.), 53-61. Singapur: Singapore Art Museum.

Fundación JuAn March (2018). El principio Asia. China, Japón e India y el arte contemporáneo en España (1957-2017). Madrid: Fundación Juan March.

HKW (1999). Gặp Việt Nam. Berlin: Haus der Kulturen der Welt.

ILLICH, I. y SANDERS, B. (2019). ABC. La alfabetización de la mente popular. Pamplona: El Pez Volador.

KoH, A. (2014). "Amanda Heng". eresources.nlb.gov.sg. Disponible en línea: https://eresources.nlb.gov.sg/infopedia/articles/SIP_2014-03-06_161502.html [26/05/2018].

KoKURITSU SHIN BiJUTSUKAn (2017). Sunshower. Contemporary Art from Southeast Asia 1980s to Now. Tokyo: Heibonsha Publishers.

LEgasPI-RAMIREZ, E. (2018). "Embodied Subjectivities: Accounts of Affect in Lani Maestro's Site-Specific Work, 1970s-1990s”. En Ambitious Alignments. New Histories of Southeast Asian Art, S. H. Whiteman, S. Abdullah, Y. Low \& P. Scott (eds.), 225-255. Singapur: Power Publications and National Gallery Singapore.

LenzI, I. (2016). "Public Play: Audience Involvement and the Decoding of Concept in Socially Engaged Southeast-Asian Contemporary Art". Obieg 2. Disponible en línea: http://obieg.u-jazdowski.pl/en/azja/public-play--audience-involvementand-the-decoding-of-concept-in-socially-engaged-southeast-asian-co [25/05/2017].

Méndez Baiges, M., ED. (2017). Arte escrita. Texto, imagen y género en el arte contemporáneo. Granada: Comares.

Morelli, N. (2019). “The Singapore Series-Chapter 13”. naimamorelli.com. Disponible en línea: http://www.naimamorelli.com/singapore-series\%e2\%80\%8a$\%$ e2\%80\%8achapter-13/[19/03/2021].

Nguyen Du. (2013). La Historia de Kieu (Kim Van Kieu) [bilingüe]. Madrid: Hiperión.

NinH, K. N. B. (2002). A World Transformed: The Politics of Culture in Revolutionary Vietnam 1945-1965. Ann Arbor: University of Michigan Press.

NuAlart, C. (2016). "Queer Art in Vietnam: From Closet to Pride in Two Decades", Palgrave Communications 2. Disponible en línea: https://www.nature.com/ articles/palcomms20169 [19/04/2016].

(2017). "La pintura a la laca en la construcción de las Bellas Artes en Vietnam", Tsantsa 5, 95-116. Disponible en línea: https://publicaciones.ucuenca.edu.ec/ ojs/index.php/tsantsa/article/view/1740 [02/05/2018].

PhAN, J. (2013). "Chữ Nôm and the Taming of the South: A Bilingual Defense for Vernacular Writing in the Chỉ Nam Ngọ Am Giải Nghĩa". Journal of Vietnamese Studies 8.1, 1-33. 
RAdUlovic, V. (2009). "Anything can happen between now and then... Performance Art in Vietnam”. En Connect: Kunstszene Vietnam, IFA (Institut für Auslandsbeziehungen), cat. exp. 87-101. Bonn: Kerber Verlag. (2012). “One Size Fits All; or, Whose Public Space?”, Guggenheim Blogs. Disponible en línea: https://www.guggenheim.org/blogs/map/one-size-fits-all-orwhose-public-space [04/03/2021].

Rawlings, A. (2010). "Where I work: Amanda Heng”. Art Asia Pacific 70. Disponible en línea: http://artasiapacific.com/Magazine/70/AmandaHeng [24/03/2020].

SABAPAthy, T. K. (1996). "Developing Regional Perspectives in South-East Asian Art Historiography". En The Second Asia-Pacific Triennial of Contemporary Art, C. Turner \& R. Devenport (eds.), 13-17. Brisbane: Queensland Art Gallery.

Scott, Ph. (2009). "Art and the Press in the Colonial Period: Phong Hóa, Ngày Nay and the École des Beaux-Arts de l'Indochine". En Essays on modern and contemporary Vietnamese Art, S. Lee \& Nguyen Nhu Huy (eds), 14-22. Singapur: Singapore Art Museum.

Steinberg, D. J., ED. (1987). In Search of Southeast Asia: A Modern History. Hawaii: University of Hawaii Press.

TAN, A. (2011). "She and Her Mother's Tongue: Touching on Amanda Heng". En Amanda Heng: Speak To Me, Walk With Me, cat. exp. 50-67. Singapur: Singapore Art Museum.

(2015). "Re:Defining Art: Art in the 1970s and after". En Between declarations and dreams: Art of Southeast Asia since the 19th century, cat. exp. 64-82. Singapur: National Gallery Singapore.

TAYLOR, N. A. (2007). "Vietnamese Anti-art and Anti-Vietnamese Artists: Experimental Performance Culture in Ha Noi's Alternative Exhibition Spaces”. Journal of Vietnamese Studies 2.2, 108-128.

(2012). "Introduction. Who Speaks for Southeast Asian Art?". En Modern and Contemporary Southeast Asian Art. An Anthology, N. A. Taylor \& B. Ly (eds), 113. Ithaca: Cornell University Southeast Asia Program Publications.

Thompson, C. M. (2010). "Signification as Limitation: Minh Mang's Prohibition on Use of Nom and the Resulting Marginalization of Nom Medical Texts". En Looking at It from Asia: The Processes that Shaped the Sources of History of Science, F. Bretelle-Establet (ed.), 393-412. Dordrecht: Springer.

VINH SINH (1995). “"Elegant Females' Re-Encountered: From Tokai Sanshi’s Kajin No Kigu to Phan Chau Trinh's Giai Nhan Ky Ngo Dien Ca". En Essays into Vietnamese Pasts, K.W. Taylor \& John K. Whitmore (eds.), 195-206, Ithaca: Cornell University Southeast Asia Program Publications.

Weng Choy LeE (2009). “Our history, large and small”. Inter-Asia Cultural Studies 10. 2, 333-339. 
Whiteman, S. H.; Abdullah, S.; Low, Y. \& ScotT, P., EDS. (2018). Ambitious Alignments. New Histories of Southeast Asian Art. Singapore: Power Publications and National Gallery Singapore.

WubIN, Zh. (2016). Photography in Southeast Asia. A Survey. Singapur: National University of Singapore Press.

El/la firmante del artículo se responsabiliza de las licencias de uso de las imágenes incluidas.

Fecha de recepción: 25/01/2021

Fecha de aceptación: 07/04/2021 